\title{
Socially aware D2D cooperative communications for enhancing Internet of Things application
}

\author{
Junjie Yan ${ }^{1}$, Dapeng Wu ${ }^{1 *}$ (D), Chenlu Zhang ${ }^{2}$, Honggang Wang ${ }^{3}$ and Ruyan Wang ${ }^{1}$
}

\begin{abstract}
To satisfy the rapidly growing requirements of wireless data services recently, fog computing which is as an extension of cloud computing is proposed for offloading the Internet of Things (IOT) data services and applications. It depends more on the collaboration of near-located devices, instead of sending the information to remote servers. In this paper, we conceive the idea of utilizing device-to-device (D2D) communications as the infrastructures for computation and communication. In particular, D2D communication technology is one of the most important and innovative revolutions in the development of future cellular networks, which greatly enlarges the system capacity and extensively enriches the service category and application diversity. In computation part, by analyzing the interest difference of users, a Virtual Fog Community (VFC) is established in this paper, which exploits the coalition game based on the transfer effectiveness to adaptively divide users into multiple fog communities. In communication part, by perceiving the importance degrees of users and accordingly employing multidimensional network status parameters such as the local centrality and closeness degree, the best cooperative user can be reasonably selected based on the physical and social attributes of users. Therefore, the corresponding inter-community and intra-community D2D cooperative transmission mechanisms are devised to dramatically optimize the network resource utilization. Simulation results show that the proposed mechanism fully exploits the social relationship between users and effectively enhances the network operational efficiency at the cost of relatively low delay.
\end{abstract}

Keywords: D2D communication, Social attributes, Internet of things

\section{Introduction}

As reported by Cisco, around 50 billion intelligent devices would be connected to the Internet for Internet of things (IoT) applications by 2020 [1]. At present, IoT applications include, e.g., smart grids and cities, wireless sensors, internet of vehicles, and so on. In such applications, IoT devices (e.g., smartphones, vehicles) often are utilized as the sink nodes to assist sensing data collection in the manner of end to end communication technology, such as device-to-device (D2D) and machine-to-machine (M2M) communications [2-5]. As the IoT devices and their applications increase tremendously, mobile data services are also growing rapidly. By 2019, the traffic of data service in

\footnotetext{
*Correspondence: wudp@cqupt.edu.cn

${ }^{1}$ College of Communication and Information Engineering, Chongqing

University of Posts and Telecommunications, Chongwen Road, Chongqing,

China

Full list of author information is available at the end of the article
}

cellular networks will be about 11 times more than that in 2014, according to the Visual Networking Index (VNI) forecasted by Cisco in February 2015. As a result, the loads of data centers and base stations grow inevitably. As to such explosive growth of mobile data services, it is becoming a global consensus that cloud computing technology is an essential driver for IoT computation speedup, energy consumption, and service realizations.

However, cloud computing provides an augment in communication but quite limited, and they are controlled primarily by network operators, which is not efficient and effective from the application aspect [6, 7]. To solve these problems, Cisco proposed a new computational paradigm, termed as fog computing, that puts a substantial amount of storage, communication, control, configuration, measurement, and management at the edge of a network, rather than establishing channels for the centralized cloud storage and utilization, which extends 
the traditional cloud computing paradigm to the network edge $[8,9]$. The architecture of fog computing utilizes a collaborative multitude of end-user clients or near-user edge devices to carry out a substantial amount of communication and computation. Due to the resource utilization of geo-related devices, fog computing has more computation capacity and less communication delay compared with the cloud computing. Therefore, fog computing is often exploited to serve large-scale IoT applications [10].

In LTE Rel-13, Narrowband IoT (NB-IoT) was introduced for cellular IoT applications [11]. The first and foremost requirement of the cellular IoT is to provide connectivity between devices. Thus, a significant endto-end communication technology in cellular networks, often termed as D2D communications, is imperative for the success of the cellular IoT. D2D communications could be also direct communication or cooperative communication. In the direct communication, devices communicate with each other via a network infrastructure, which could be an access point or a base station. For cooperative communication, devices cooperative information for each other achieve end-to-end communication between any source and destination device [12-16]

What is more, in D2D cooperative communication scenarios, users participate and construct a complex social networks by exploiting social attributes of users, such as interests in common, users background, and so on. Numerous study results pointed out the "big world, small world" feature of mobile networks [17]. In the "small world," users encounter with each other frequently and the encounter duration is relatively long, whereas users rarely encounter with each other in the "big world." Such social characteristics can be applied by fog computing, and then users can be divided into different social groups, namely, Virtual Fog Community (VFC). VFC can makes full use of local user information to serve the requirements of some specific IoT applications such as some delay tolerant aware services at a lower transmission cost [18]. Therefore, fog computing combining the social attributes of users and D2D cooperative communication is an important research direction in the cellular IoT.

In recent years, social attributes of users are explored in D2D cooperative communication [19]. A series of social relationship structures and social phenomena were analyzed by Ref. [20, 21], such as the definition of community and closeness, which further concludes that the stable social structure and social relationship exist in D2D networks. The very first application of social attributes in D2D communication was the social attribute aware D2D network framework proposed in Ref. [22], which preliminarily analyzed the social relationship between the network structure and users. Compared with the D2D communication methods without considering social attributes and the direct transmission, it can bring significant network performance improvements. The relationship between content requirements and historical contact information of users was analyzed in Ref. [23]. To effectively reduce the base station load through D2D links, a contact time based D2D resource allocation algorithm was proposed in Ref. [24] to comprehensively consider the encounter time and contact time, by which sociality-aware and sociality-blind resource allocation algorithms are compared. Although the abovementioned references exploited social attributes to analyze D2D data transmission, D2D users are moving in social networks and their trajectories are regular, which were not pointedly analyzed and studied by them. Therefore, the social relationship between users such as the horizontal and vertical relationships cannot be fully exploited in fog computing to achieve the efficient data forwarding, where the horizontal and vertical relationships describe users with similar behaviors and users with different characteristics respectively.

To solve these problems, in computation part, VFC is proposed based on D2D communications in this paper. In particular, the network structure is firstly detected according to the interest spots difference between users, upon which a physical and social attribute Cooperative Aware Data Forwarding Mechanism (CADFM) is designed in communication part. CADFM extensively considers and reasonably exploits the social relationships between intra-community and inter-community users to select the optimal cooperative user. The main contributions of this paper can be summarized as follows.

- VFC is proposed in this paper to quantify the user interest degree for various spots, according to the user trajectory regularity. By analyzing the in-network user interest difference and through the coalition game, users are adaptively divided into fog communities, which make the network architecture strongly scalable and suitable for various complex network scenarios.

- A user importance evaluation method is proposed in this paper to estimate the local centrality and closeness degree of users, which indicate the horizontal social relationship, namely the intra-community social relationship, and vertical social relationship, namely inter-community social relationship. As a result, the intra-community and inter-community data transmission efficiency can be effectively enhanced.

- A physical and social attribute cooperative aware data forwarding mechanism is proposed in this paper to fully exploit the social relationship between intra-community and inter-community users. By adaptively perceiving the network status, the 
cooperative users can be reasonably selected, which achieves the efficient data forwarding while reducing the average delay.

The rest of this paper is organized as follows. A comprehensive outline of the fog computing architecture is proposed based on D2D communications in Section 2, and the user interest difference perceiving and VFC construction methods are further proposed in Section 3. Section 4 evaluates the transmission rate between users. Section 5 describes the user importance perceiving mechanism, whereas a social and physical attribute cooperatively perceiving D2D data forwarding mechanism is introduced in Section 6. Furthermore, the settings of simulation scenarios are given and the performance of the proposed algorithm is analyzed in Section 7. Lastly, the conclusion is given in Section 8.

\section{Network architecture}

Based on the D2D direct communication or cooperative communication, the evolved fog architecture is shown in Fig. 1.

Fog architecture includes three layers: the first layer (device layer) encompasses all the sensing devices, which are responsible for transmitting the sensing data to other sensing devices or its immediate upper layer in the hierarchy; the second layer (access layer) includes evolved access networks and logic fog computing layer. To incorporate fog computing in edge devices, the evolved access network contains the traditional remote radio heads (RRH) and the fog-computing-based access point (F-AP). What is more, the logic fog computing layer is introduced to manage some distributed communication and storage functions; the third layer (cloud computing network layer) is the upper-most layer in this architecture. The layer

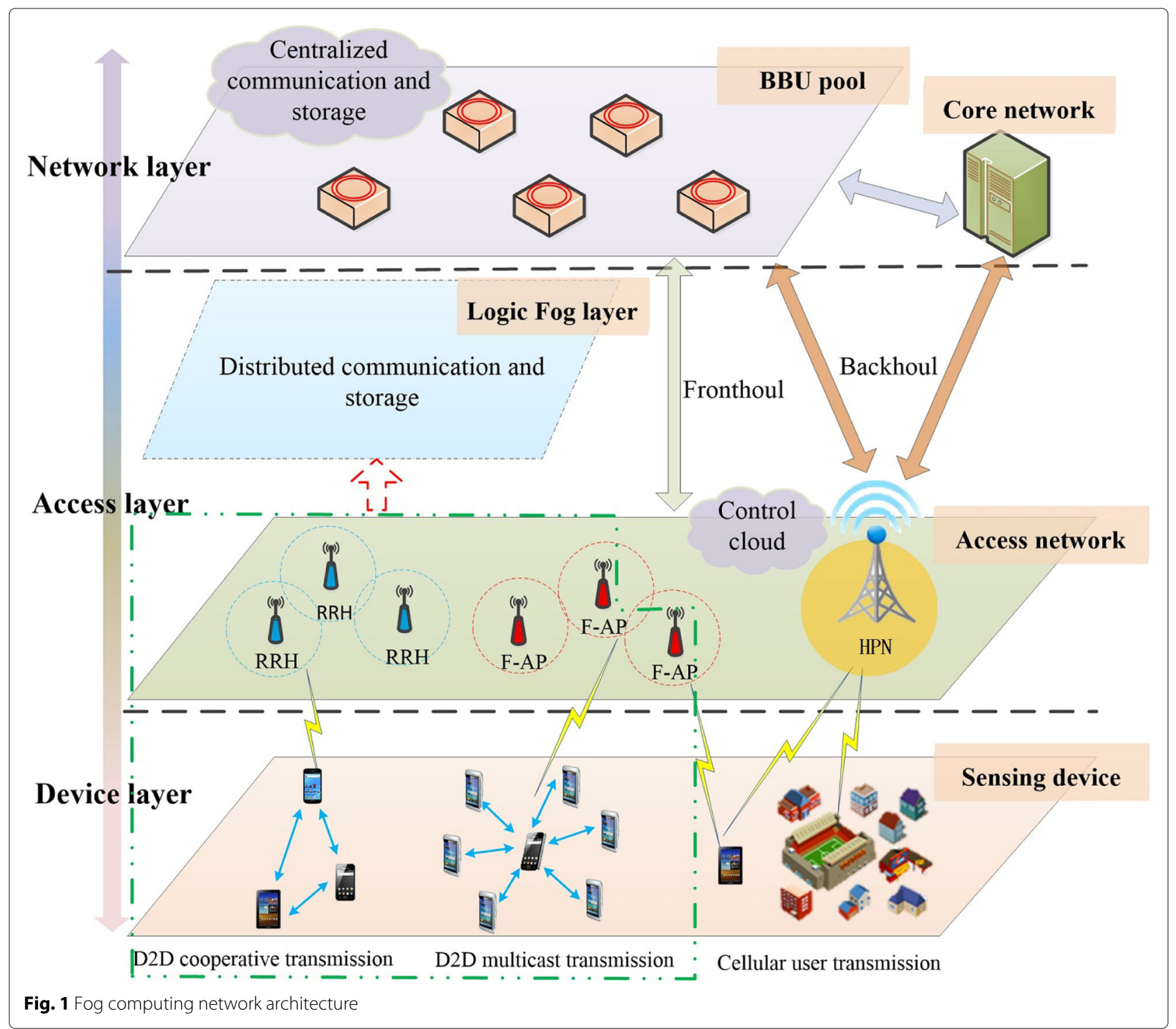


constitutes of multiple high-end servers and data centers which are capable of processing and storing an enormous amount of data.

\section{VFC building}

As mentioned above, the logic fog computing layer is responsible for local distributed communication by $\mathrm{D} 2 \mathrm{D}$ communications. However, users are strongly socially related in D2D networks. Therefore, making full use of social attributes can be effective to assist logic fog computing layer in managing the distributed communication. In this section, a logical structure detection method is firstly proposed based on user interest spots difference. The behaviors, encounter probability, and encounter duration of users within the same fog community are similar. Besides, the user interest for a given spot may change as network operates and the interest difference may appear for physically close users. Therefore, determining cooperative user solely according to the physical attributes may not be reasonable, and the inaccurately predicted connection durations of temporary links and encounter probability may not meet the requirements of requested resources. Besides the physical attributes, social attributes are considered also for a reasonable selecting of cooperative users $[25,26]$.

\subsection{Interest difference perceiving}

The user activities are regular, and social relationship between them is relatively stable. To effectively describe the regularity of user behaviors, the appearance times at various interest spots are recorded by the base station, by which the interest matrix $I$ of a given user can be acquired. By comparing the current interest matrices of users, their current interest difference can be derived, where a small user interest difference means the possibly similar user trajectories and behaviors. By exploiting these similarities, the network can be divided into logical components, namely VFC for the further design of a more efficient data forwarding mechanism.

Assuming there are $n$ users in the network, $U=$ $\left\{u_{1}, u_{2}, \cdots, u_{n}\right\}$ respectively which can be recorded by $\mathrm{BS}$, and user $u_{i}$ has $m_{i}$ interest spots, $M_{u_{i}}=$ $\left(a_{u_{i}}^{1}, a_{u_{i}}^{2}, a_{u_{i}}^{3} \cdots a_{u_{i}}^{m_{i}}\right)$ respectively, and interest spots of different users may overlap. The complete set of interest spots can be obtained according to Eq. (1), where $b_{i} \cap b_{j}=$ $\emptyset, i \neq j ; i, j=1,2, \cdots, p$.

$$
B_{i}=M_{u_{1}} \cup M_{u_{2}} \cup M_{u_{3}} \cdots \cup M_{u_{n}}=\left\{b_{1}, b_{2}, b_{3}, \cdots, b_{p}\right\}
$$

By recording the appearance times at various interest spots of a given user in a given period, the interest degree of users can be estimated for a certain interest spot. The larger appearance times clearly indicate a higher interest degree for a certain interest spot. By comparing the proportions of the appearance times at a given spot to the total appearance times, the interest degree for all spots of user $u_{i}$ in time $t$ are $F_{u_{i}}(t)=$ $\left(f_{u_{i}}^{b_{1}}(t), f_{u_{i}}^{b_{2}}(t), f_{u_{i}}^{b_{3}}(t) \cdots, f_{u_{i}}^{b_{p}}(t)\right)$, where constraints hold as shown in Eqs. (2) and (3).

$$
\begin{aligned}
& 0 \leq f_{u_{i}}^{b_{z}}(t) \leq 1(1 \leq z \leq p) \\
& \sum_{z=1}^{p} f_{u_{i}}^{b_{z}}=1
\end{aligned}
$$

where $f_{u_{i}}^{b_{z}}(t)$ denotes the interest degree for spot $b_{z}$ of user $u_{i}$ in time $t$. Eventually, the interest matrix $I=$ $\left\{F_{u_{1}}(t), F_{u_{2}}(t), \cdots F_{u_{n}}(t)\right\}^{T}$ can be obtained.

Definition 1 (User Interest Difference). At time $t$, the interest of user $u_{x}$ and $u_{y}$ for given spot $b_{z}$, namely $f_{u_{x}}^{b_{z}}(t)$ and $f_{u_{y}}^{b_{z}}(t)$ are different. Their current interest matrices are analyzed and compared to define the interest difference between user $u_{x}$ and $u_{y}$ as shown in Eq. (4), by averaging their interest difference for all spots.

$$
D\left(u_{x}, u_{y}\right)= \begin{cases}0 & \\ \sum_{z=1}^{p} \frac{\left|f_{u_{x}}^{b_{z}}(t)-f_{u_{y}}^{b_{z}}(t)\right|}{p} & F_{u_{x}}(t)=F_{u_{y}}(t) \\ & \text { others }\end{cases}
$$

As shown in Eq. (4), $D\left(u_{x}, u_{y}\right)=0$ denotes that there is no interest difference between user $u_{x}$ and $u_{y}$, whereas $D\left(u_{x}, u_{y}\right)=1$ indicates that two users share no interest spots.

\subsection{Fog community detecting}

To meet the dynamic features of the network scenario, the minimum overall interest difference of communities is employed as the objective function. In order to achieve this goal, users are adaptively assigned to fog communities. Obviously, constructing the objective function of minimum fog community difference is crucial to optimize the fog community detection in this paper. Therefore, the coalition game model is employed to describe the relationship between users in a given fog community to minimize the fog community difference, to further exploit the stable social relationship, and to accordingly enhance the data forwarding efficiency.

\subsubsection{The establishment of game coalition and objective function}

During initialization, if $\max F_{u_{i}}(t)=f_{u_{i}}^{b_{x}}(t)$ at time $t$, $u_{x}$ is arranged into fog community $b_{x}$. Further, the current fog community set can be defined as $S^{\prime}=$ $\left\{s_{1}, s_{2}, s_{3}, \cdots, s_{v}\right\}(v \leq p)$, namely the game coalition, 
where $s_{i} \cap s_{j}=\emptyset(i \neq j ; i, j=1,2, \cdots, p)$ and the coalition participants are $U=\left\{u_{1}, u_{2}, \cdots, u_{n}\right\}$ respectively (normally $v \leq n)$. Assuming the $s_{k}$ th $(1 \leq k \leq v)$ fog community has $\theta_{s_{k}}$ users $\left(1 \leq \theta_{s_{k}} \leq n\right)$ and the sum of fog community members is defined by Eq. (5).

$$
\sum_{k=1}^{v} \theta_{s_{k}}=n
$$

However, even when users share the same interest spot, their interest may be different. Some users may have the same level of interest for all spots, whereas other users may have stronger interest for one or several spots among all their interest spots. To minimize the interest difference within each fog community, users may reasonably joint or quit given fog communities, namely user transferring.

$$
\Omega_{s_{k}}=\frac{\sum_{u_{x}=1}^{\theta_{s_{k}}} \sum_{u_{y}=1}^{\theta_{s_{k}}} D\left(u_{x}, u_{y}\right)}{\theta_{s_{k}}^{2}}
$$

where $\Omega_{s_{k}}$ represents the interest difference between each other in fog communities $s_{k}$. Because the alteration of belonging fog community can cause the change of fog community difference, the user transfer effectiveness is set to the fog community difference. As mentioned above, to reasonably transfer users, the overall interest difference of communities, namely the objective function, should be minimized, as shown in Eq. (7).

$$
J=\min \sum_{k=1}^{v} \Omega_{s_{k}}
$$

\subsubsection{Fog user transferring}

Targeting at the abovementioned problems, the adaptive preference order adjustment method in coalition game is employed to assign the optimal fog communities for each user, namely the user transferring [27], by which the interest difference within each fog community can be minimized and a temporarily stable status of a given fog community can be achieved.

Assuming user $u_{i}$ is initially arranged into fog community $s_{k}$, when $u_{i}$ prefers the new fog community $s_{l}$ than the original fog community $s_{k}$, this situation is denoted by $s_{l} \succ{ }_{u_{i}} s_{k}$. The user transferring constraints are shown in Fig. 2, where the user can be transferred if and only if the interest difference of original fog community $s_{k}$ does not increase and the interest difference of new fog community $s_{l}$ decreases.

$$
\Omega_{s_{l}}>\Omega_{s_{l^{\prime}}} \text { and } \Omega_{s_{k}} \geq \Omega_{\left(s_{k} \backslash i\right)}
$$

In Eq. (8), $s_{l^{\prime}}$ is the new fog community of user $u_{i}$ and $\left(s_{k} \backslash i\right)$ is the original fog community. By repeating the user

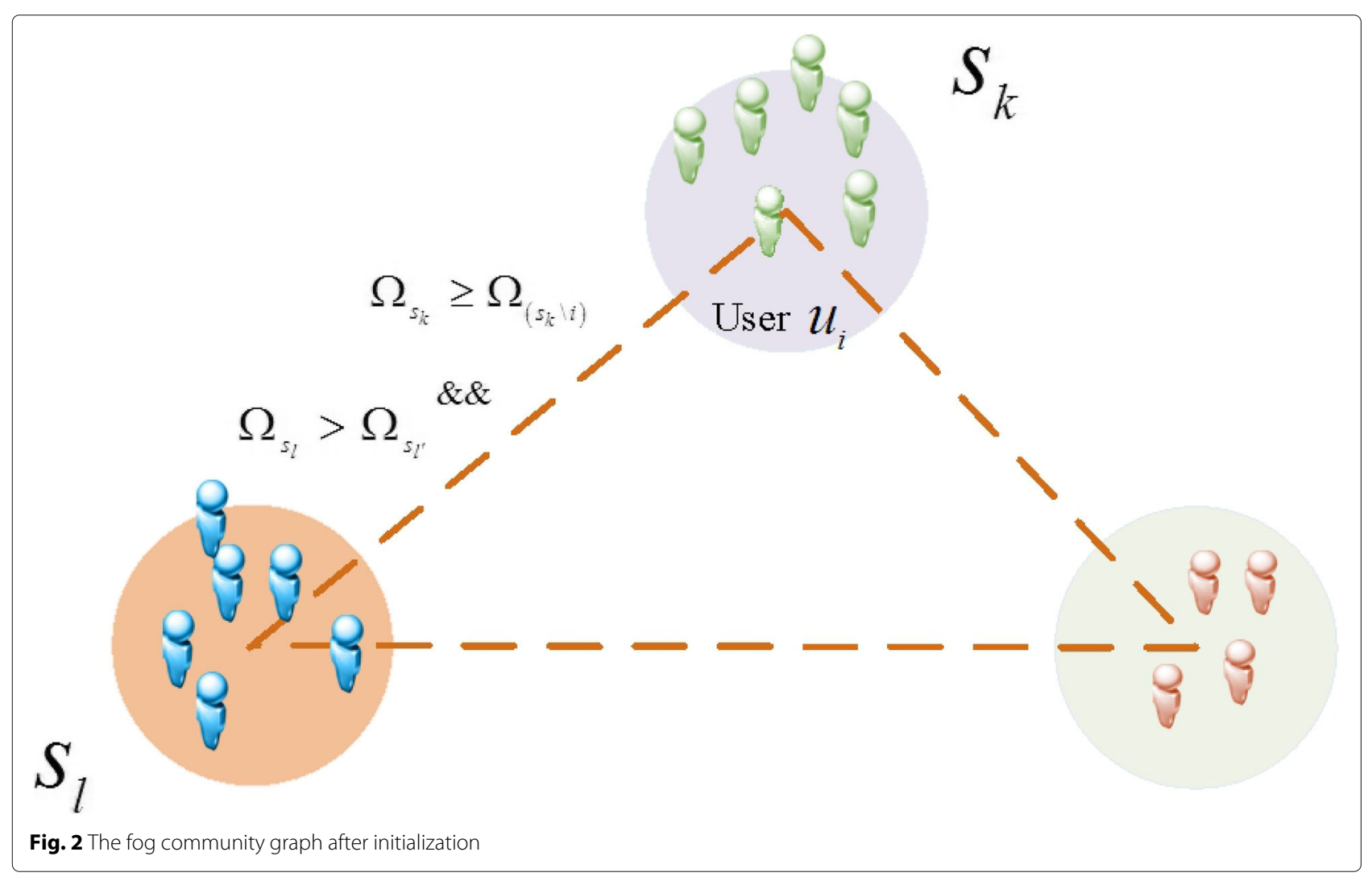


transferring constraints on all users until Nash equilibrium is achieved, the value of objective function can be minimized.

\subsubsection{Fog community updating}

Due to the time variant characteristic of user interest and the randomness of user movements, the interest of a given user is dynamic and their difference also changes, which has to be dynamically updated to evaluate and reflect the user relationship. To solve this problem, staying time of user $u_{i}$ is defined in this paper, which is determined by the interest difference between $u_{i}$ and other users in this fog community. Obviously, for user $u_{i}\left(u_{i} \in s_{k}\right)$, its interest difference from other users in $s_{k}$ is defined by Eq. (9).

$$
\Omega_{s_{k}}^{u_{i}}=\frac{\sum_{u_{x}=1}^{\theta_{s_{k}}} D\left(u_{i}, u_{x}\right)}{\theta_{s_{k}}}
$$

According to Eq. (6), $0<D\left(u_{i}, u_{x}\right)<1$; therefore, $0<$ $\Omega_{s_{k}}^{u_{i}}<1$. Assuming the maximum staying time is $T_{s}$, the staying time of user $u_{i}$ in $s_{k}$ can be denoted by Eq. (10).

$$
\Delta_{s_{k}}^{u_{i}}=T_{s}\left(1-\Omega_{s_{k}}^{u_{i}}\right)
$$

At time $t+\Delta_{u_{i}}^{s_{k}}$, the fog community set of user $u_{i}$ is updated. Besides, the user transferring constraints are evaluated to determine which fog community for user $u_{i}$ to join and the corresponding staying time.

As can be seen, the relationship between users within the same fog community is close and they encounter frequently according to the adaptive logical structure detection results. Contrarily, users from different fog communities rarely encounter. Therefore, the horizontal and vertical social relationship between users can be more accurately evaluated after the logical structure detection, and the data forwarding decision can be reasonably achieved while guaranteeing high data transmission efficiency and delivery ratio.

Due to the difference of the user density and velocity in the network, physical attributes also affect the selecting of cooperative users. For instance, when the user distribution is sparse, the social relationship between users is insignificant. Therefore, physical attributes such as the transmission rate contribute majorly to the cooperative user selecting decision. Besides, when the user velocity is high, the encounter duration between users is relatively small and not enough for the data transmission process. Therefore, to enhance the data transmission efficiency, the user velocity is another factor that determines the cooperative user selecting.

\section{Sensing data transmission model}

In the underlay mode, D2D communication and cellular communication are both available for users. If user $u_{i}, u_{j} \in U$ are communicating through D2D and sharing the uplink channel of user $u$, their communication can be interfered by cellular user $u[28,29]$. In various network scenarios, physical attributes of users have considerable impacts on the network performance. Assuming $P_{u_{i}}$ is the transmitting power of D2D user $u_{i}$, the channel gain between user $u_{i}$ and $u_{j}$ is $h_{u_{i}, u_{j}}$; the received signal of user $u_{i}$ can be denoted by $P_{u_{i}} h_{u_{i}, u_{j}}$. To be general, channels are assumed to follow Rayleigh distribution, the distance between users $u_{i}$ and $u_{j}$ is denoted by $d_{u_{i}, u_{j}}$, and the channel attenuation factor and Gaussian channel coefficient are denoted by $\partial$ and $h_{0}$ respectively. Because the communication between users is under the impact of their distance and channel attenuation, the channel gain between them can be denoted by

$$
h_{u_{i}, u_{j}}=d_{u_{i}, u_{j}}^{-\partial} h_{0}
$$

The noise of the received signal is composed of the additive Gaussian white noise $N_{0}$ [30], and the same frequency interference noise by cellular user $u$. Therefore, the noise of the received signal by user $u_{i}$ can be denoted by $P_{u} h_{u, u_{j}}+N_{0}$. Furthermore, according to the Shannon equation, the transmission rate between D2D users $R_{u_{i}, u_{j}}$ can be denoted

$$
R_{u_{i}, u_{j}}=\log _{2}\left(1+\frac{P_{u_{i}} h_{u_{i}, u_{j}}}{P_{u} h_{u, u_{j}}+N_{0}}\right)
$$

D2D transmission rate is estimated in this section to further consider the physical attribute for a reasonable cooperative user selection. However, only considering the user physical attributes may cause inaccuracy; thus, the horizontal and vertical social relationships should be taken into account. By perceiving the user centrality, the social relationship between users can be reasonably evaluated and the social attributes can be fully exploited, which can be combined with the physical attributes to determine the optimal cooperative user and to achieve the efficient data forwarding.

\section{User importance perceving}

The stable social relationship between users can be exploited to enhance the data forwarding efficiency in D2D cooperative communication scenarios. Because users from the same fog community are all socially close, the local centrality which describes the relationship among users within the same fog community will be used for user importance of same fog community, while the relationship of users from the different fog community is not all closed. Therefore, the closeness degree which describes the encounter frequency between two users with different fog community is used for user importance of different fog community. By analyzing the proposed local centrality and closeness degree between users, user 
importance between users from the same and different fog community can be evaluated.

\subsection{Local centrality}

According to its definition, the local centrality only focuses on users from the same fog community and only evaluates the directly related users. Therefore, the estimation of connection times should also consider the connection duration, and only the connection long enough for the data transmission can be regarded as effective. For user $u_{i}$, Eq. (11) describes the encounter between it and user $u_{j}$.

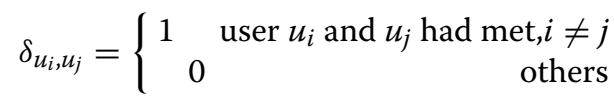

To avoid the impact of these invalid short connections, Eq. (12) gives the improved connection determination method by considering the encounter duration.

$$
\eta_{u_{i}, u_{j}}=\left\{\begin{array}{rr}
1 & \text { duration }\left(u_{i} u_{j}\right) \geq t_{u_{i}, u_{j}}, i \neq j \\
0 & \text { others }
\end{array}\right.
$$

In Eq. (12), duration $\left(u_{i}, u_{j}\right)$ denotes the encounter duration of user $u_{i}$ and $u_{j}$, and $t_{u_{i}, u_{j}}$ denotes the total transmission delay between user $u_{i}$ and $u_{j}$, which is related to the data transmission rate $R_{u_{i}, u_{j}}$ and data size $F$, as shown in Eq. (15).

$$
t_{u_{i}, u_{j}}=\frac{F \cdot \operatorname{Ret}}{R_{u_{i}, u_{j}}}+\frac{d_{u_{i}, u_{j}}}{c}
$$

In Eq. (13), $F$ denotes the data size and Ret denotes the retransmission times. $c$ is the light speed. Obviously, the direct social relationship exists only if Eqs. (12) and (13) are met. Therefore, the local centrality, $\xi_{u_{i}}$ of user $u_{i}$ in community $s_{k}$ can be defined by Eq. (14).

$$
\xi_{u_{i}}=\frac{\sum_{j=i}^{\theta_{s_{k}}} \delta_{u_{i}, u_{j}} \cap \sum_{j=i}^{\theta_{s_{k}}} \eta_{u_{i}, u_{j}}}{\theta_{s_{k}}}
$$

As can be seen, Fig. 3 describes the intra-community logical relationship after the local centrality analysis. For instance, user $u_{A}$ has five directly related users and the size of community is 16 , its centrality can be calculated according to Eq. (16). To be more specific, given a community with 100 users, if a user has 25 directly related users, its local centrality is 0.25 . Due to various community sizes, the local centrality can be only utilized to compare users from the same fog community.

\subsection{Closeness degree}

To estimate the closeness degree between users, the encounter frequency is further analyzed and employed in this paper, by which the date can rapidly transmitted to

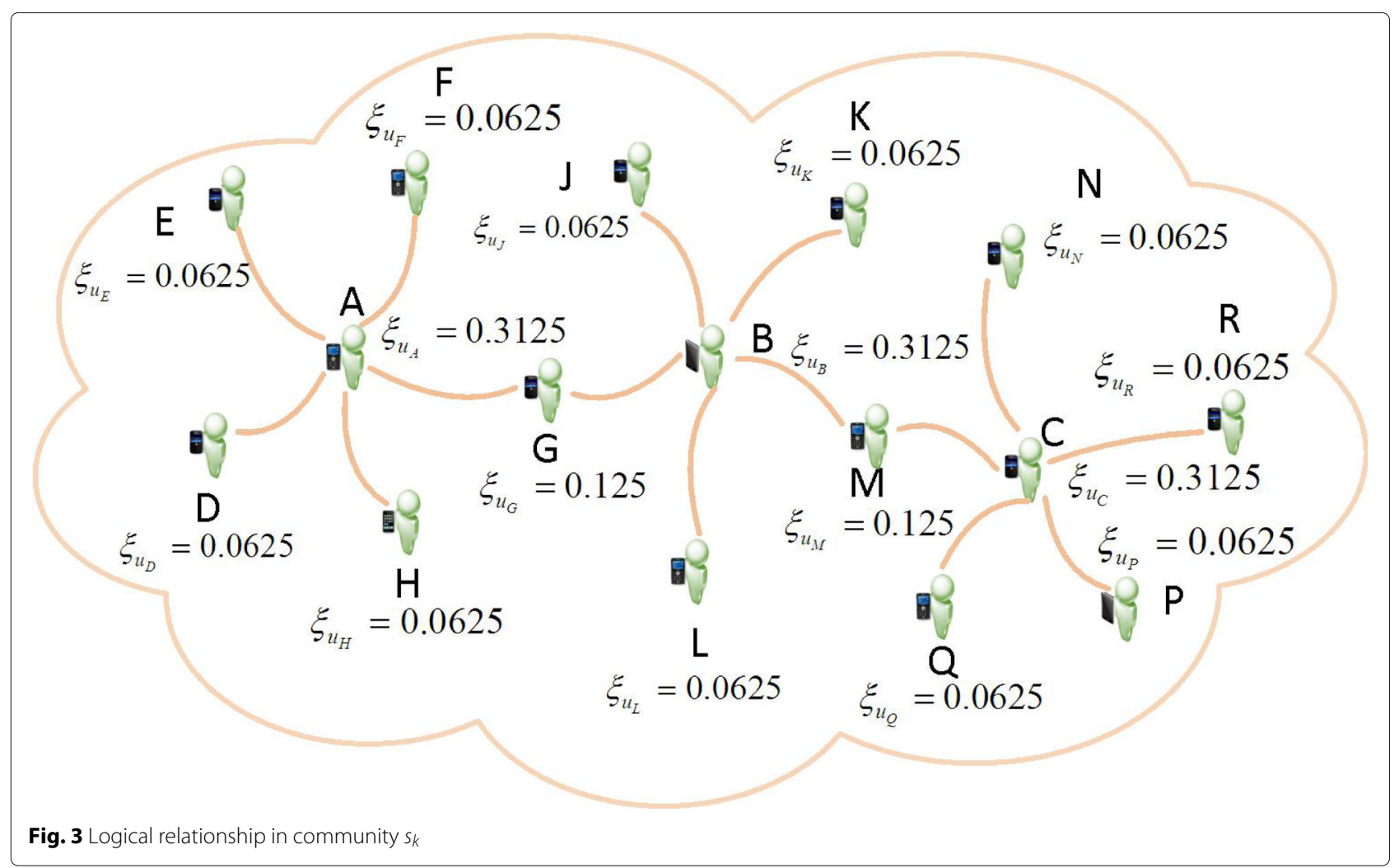


the receiving user. According to the user mobility analysis given by Ref. [31, 32], the encounter interval between users can be described by the Pareto distribution. We assume that $T_{u_{i}, u_{j}}$ denote the encounter interval between user $u_{i}$ and $u_{j}$. The complementary cumulative distribution function of $T_{u_{i}, u_{j}}$ can be calculated by Eq. (17).

$$
P_{u_{i}, u_{j}}\left\{T_{u_{i}, u_{j}}>t\right\}=\left(\frac{\tau_{u_{i}, u_{j}}^{\min }}{t}\right)^{\alpha_{u_{i}, u_{j}}}, t \geq \tau_{u_{i}, u_{j}}^{\min }
$$

where the $\alpha_{u_{i}, u_{j}}>0$ determines the shape of $T_{u_{i}, u_{j}}$ 's probability distribution function and $\tau_{u_{i}, u_{j}}^{\min }$ denotes the minimum possible value of $T_{u_{i}, u_{j}}$.

Thus, the closeness degree between user $u_{i}$ and $u_{j}$ during the period $\Delta t$ can be calculated by Eq. (18).

$$
p_{u_{i}, u_{j}}(\Delta t)=1-\left(\frac{\tau_{u_{i}, u_{j}}^{\min }}{\Delta t}\right)^{\alpha_{u_{i}, u_{j}}}
$$

In this section, by evaluating the local and global centralities, two important factors are provided for the cooperative user selecting of fog computing, where local centrality describes the relationship between locally directly related users and is employed to compare users from the same community, and the global centrality describes the active degree of a given user and is utilized to reasonably select the rapid cooperative user for inter-community data forwarding.

\section{Physical and social attribute cooperative aware data forwarding mechanism}

As is mentioned above, the data transmission between users can be divided into the intra-community and intercommunity data forwarding. Due to the social relationship difference between physically close neighbor users, the temporarily established links without the social relationship consideration may be invalid short connections, which may cause the low resource utilization. Therefore, the cooperative user selecting should comprehensively consider the physical attributes along with the social attributes, by which the optimal cooperative user can be selected for the reasonable intra-community and intercommunity D2D cooperative communications so as to achieve the low delay.

\subsection{Intra-community D2D cooperative communications}

Users from the same community are socially close, encounter each other with high probability, and have stable links and long encounter duration. With more directly related users, the local centrality of the given user is higher, which can be regarded as the factor to select the intra-community cooperative user. Besides, employing the transmission rate between users also contributes to the forwarding efficiency. Therefore, the cooperative intra-community cooperative user selecting strategy is proposed in this section. The importance degree of cooperative users can be calculated by Eq. (20), where $u_{x}\left(0 \leq x \leq \theta_{s_{k}}, i \neq x\right)$ cooperative user candidates within the communication range of sending user $u_{i} \in s_{k}$ and receiving user.

$$
\operatorname{Em}(i, x)=\alpha \xi_{u_{x}}+(1-\alpha) R_{u_{i}, u_{x}}
$$

In Eq. (19), $\alpha$ is the weight factor, which is obviously crucial for the accurate selecting of the optimal cooperative user. Besides, physical attributes such as the user density and velocity within the given community also affect the intra-community D2D cooperative communications. For instance, the user density obviously affects the number of directly related users and contributes to the local centrality. Besides, in the scenario of high user velocities, data have to be rapidly forwarded to cooperative users before connections end, which signifies the importance of transmission rates. In conclusion, the weight factor $\alpha$ should be directly proportional to the user density and inversely proportional to the user velocity, as shown in Eq. (19).

$$
\alpha=1-\exp \left(-\frac{\theta_{k}}{n V_{i}}\right)
$$

where $V_{i}$ denotes the movement velocity of user $u_{i}$ and the user with the maximum $\operatorname{Em}(i, x)$ within the communication range of user $u_{i}$ is selected as the optimal intra-community cooperative user.

\subsection{Inter-community D2D cooperative communications}

When sending and receiving users share no same fog community, the inter-community data forwarding is necessary. Because sending user may not have even remote relationship with the destination community, data should be rapidly forwarded to the user who have the highest closeness degree with receiving users, assuming users, in the communication range of the sending user and receiving user, have the highest encounter probability with receiving users. Because sending and receiving users share no same fog community, the user with the highest encounter probability with the receiving user is selected to forward the data and to minimize the transmission delay. For cooperative user $u_{x}$, the encounter probability $p_{u_{x}, u_{j}}(t)$ between users can be calculated by Eq. (18).

The pseudo code of proposed CADFM is as follows, and the computational complexity of the proposed algorithms is $\mathrm{O}(n)$.

\section{Numerical results}

We perform an analysis of the fog computing paradigm combining the social attributes of users. Simulation results validate the performance of CADFM in the actual 


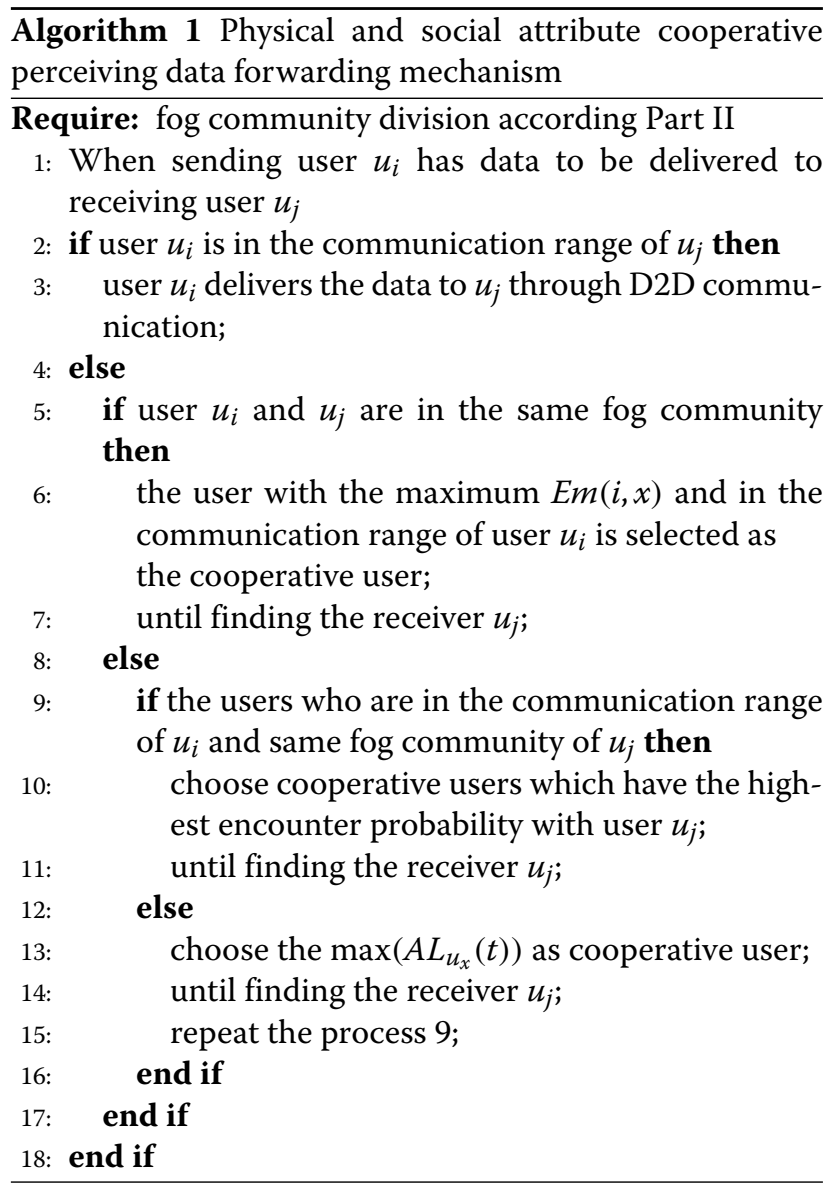

measurement scenario of social network. By fully exploiting the physical and social attributes of users, the intercommunity and intra-community data forwarding can be reasonably achieved to finally realize the efficient data delivery.

In this part, we used human mobility trace Infocom06, which is a real user mobility trace. This trace is collected by the Cambridge Haggle projects [33]. For the Infocom06, it records 98 iMotes' contact during the Conference IEEE Infocom 2006 among which there are 20 static iMotes and 78 mobile iMotes. In our simulation, we use the 20 static iMotes as interest spots and 78 mobile iMotes as mobile users. Other simulation parameters are as follows:

To reflect the performance gain of the proposed CADFM, two typical data forwarding mechanisms, Encounter Probability Data Forwarding Mechanism (EPDFM) and Direct Delivery Mechanism (DDM), are compared during the simulation, where EPDFM selects the user with the highest encounter probability to the receiving user as the cooperative user and DDM only forwards data when the receiving user is in the communication range of the sending user. Three performance metrics are compared in this paper, such as the successful
Table 1 Simulation parameters

\begin{tabular}{ll}
\hline Parameter & Value \\
\hline Carrier frequency & $2.5 \mathrm{GHz}$ \\
System bandwidth & $5 \mathrm{MHz}$ \\
eNodeB Tx power & $46 \mathrm{dBm}$ \\
Maximum UE Tx power & $29 \mathrm{dBm}(\mathrm{D} 2 \mathrm{D}$ mode: $20 \mathrm{dBm})$ \\
RB size & $12 \mathrm{sub}-$ carriers $0.5 \mathrm{~ms}$ \\
Path loss (cell link) & $128.1+37.6 \log (\mathrm{d}), \mathrm{d}[\mathrm{km}]$ \\
Path loss (D2D link) & $40 \log (\mathrm{d})+30 \log (\mathrm{f})+49, \mathrm{~d}[\mathrm{~km}], \mathrm{f}[\mathrm{Hz}]$ \\
Cell radius & $500 \mathrm{~m}$ \\
The maximum distance (D2D) & $50 \mathrm{~m}$ \\
Thermal noise & $-174 \mathrm{dBm} / \mathrm{Hz}$ \\
\hline
\end{tabular}

delivery ratio and delivery delay, and they are defined as follows.

The successful delivery ratio is defined as the proportion of the number of delivered data to that of generated data, as shown in Eq. (22).

$$
D r_{\text {del }}=\frac{D a_{\text {del }}}{D a_{\text {tot }}}
$$

The average delivery delay is defined by Eq. (23), where $\left\{t_{\text {sta } 1}, t_{\text {sta } 2}, \cdots, t_{\text {sta } \lambda}\right\}$ and $\left\{t_{\text {end } 1}, t_{\text {end } 2}, \cdots, t_{\text {end } \lambda}\right\}$ denote the data generating time and delivering time respectively.

$$
D e_{a v}=E\left\{t_{\mathrm{end} 1}-t_{\mathrm{sta} 1}, \cdots, t_{\mathrm{end} \lambda}-t_{\mathrm{sta} \lambda}\right\}
$$

7.1 Network performance under various number of users In this section, we assume that the time of simulation is $12 \mathrm{~h}$, the time to live (TTL) of data is $12 \mathrm{~min}$ and the data generating interval follows exponential distribution within the range of 50-100 s. The numbers of users ranges from 28 to 78. The performance of CADFM is compared with other two mechanisms through simulation.

The successful delivery ratio directly reflects the reliability of data transmission. As shown in Fig. 4, the increased number of users leads to the increased network user density, which affects the connection times and accordingly the data delivery ratio. Because CADFM employs the reasonable fog community detection and the optimal cooperative user is selected according to the social attributes of users, the delivery ratio of CADFM is higher than those of EPDFM and DDM. However, the users are always resource limited; thus, the active users forward most of data, and congestions at these active users prevent the further increase of delivery ratio. According to simulation results, CADFM can achieve 10.2 and $47.6 \%$ higher delivery ratios, respectively, when compared with EPDFM and DDM.

The average delivery delay under various numbers of users is shown in Fig. 5. The average delivery delays 


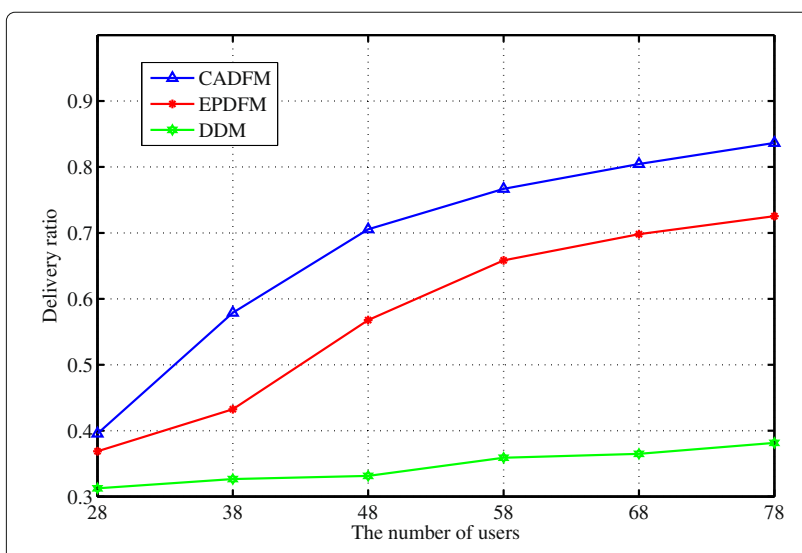

Fig. 4 The successful delivery ratio under various numbers of users

of all three mechanisms are reduced because of the increased number of users and user encounter probability. For DDM, the difficulty of reaching the receiving user is increased due to the growing number of users; thus, the average delivery delay is the highest. As shown in Fig. 5, the average delivery delay of CADFM is the lowest, that of EPDFM is higher, because the proposed cooperative selecting process is based on the centralities and encounter probabilities and the data forwarding is more reasonable, accurate, and rapid. Simulation results show the proposed CADFM can achieve 14.2 and $45.2 \%$ lower average delivery delays, respectively, when compared with EPDFM and DDM.

\subsection{Network performance under various TTL of data}

In this section, we assume that the time of simulation is $12 \mathrm{~h}$, the number of users is 78 , and the data generating interval also follows exponential distribution within the range of $50-100 \mathrm{~s}$. The TTL ranges from 3 to $12 \mathrm{~min}$. We compared the performance of CADFM with other two mechanisms under various TTL of data.

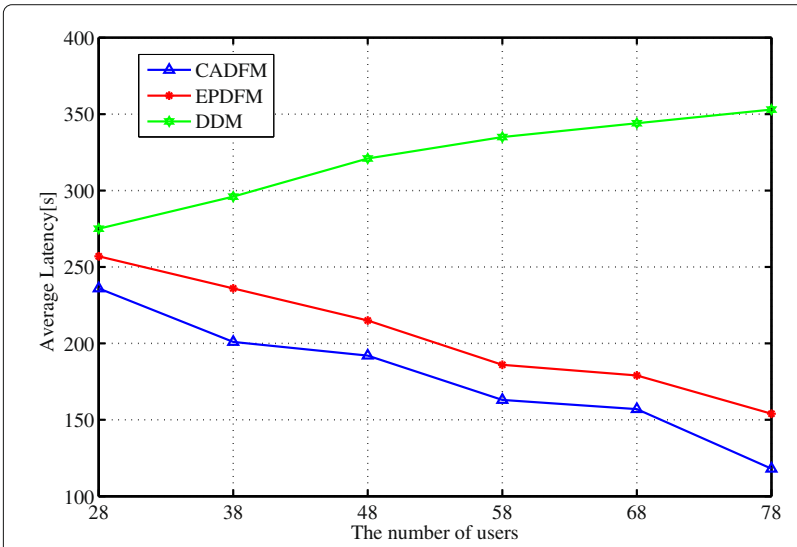

Fig. 5 The average delivery delay under various numbers of users
The successful delivery ratios under various TTL of data are shown in Fig. 6. As can be seen from the results, the delivery ratios of three data forwarding mechanisms increase along with the growing TTL of data. The main reason is that the data carrying time by a cooperative user is extended due to the growing TTL and the forwarding times also increases, which leads to the increase of the successful delivery ratio. According to simulation results, compared with EPDFM and DDM, CADFM can achieve 11.21 and $38.3 \%$ higher delivery ratios respectively. Furthermore, with the increasing TTL, the delivery ratio trend of CADFM changes obviously, while the other two mechanisms without the social attribute consideration have insignificant performance gains, due to their randomness of cooperative user selecting strategy.

The average delivery delays under various TTL of data for three mechanisms are shown in Fig. 7. As can be seen, the average delivery delay of EPDFM is lower than DDM, and the average delivery delay of DDM is the highest. Due to the inaccurate cooperative selecting strategy and the increased data carry time and forwarding times, the average data delivery delay of DDM notably increases. While fully exploiting social attributes of users, the data delivery delay of CADFM is the lowest, which can clearly reflect the importance of social attributes to the delay performance in fog computing.

\section{Conclusions}

In this paper, we proposed a novel approach CADFM for social D2D networks to achieve the efficient data forwarding and reliable data delivery. By analyzing the instantaneous user interest difference, the VFC can be detected for the further realization of the efficient data forwarding. Simulation results show that, compared with traditional fog computing data forwarding mechanisms EPDFM and DDM, the proposed CADFM can achieve the more efficient data forwarding at the cost of a relatively low delivery delay. Future work includes applying some

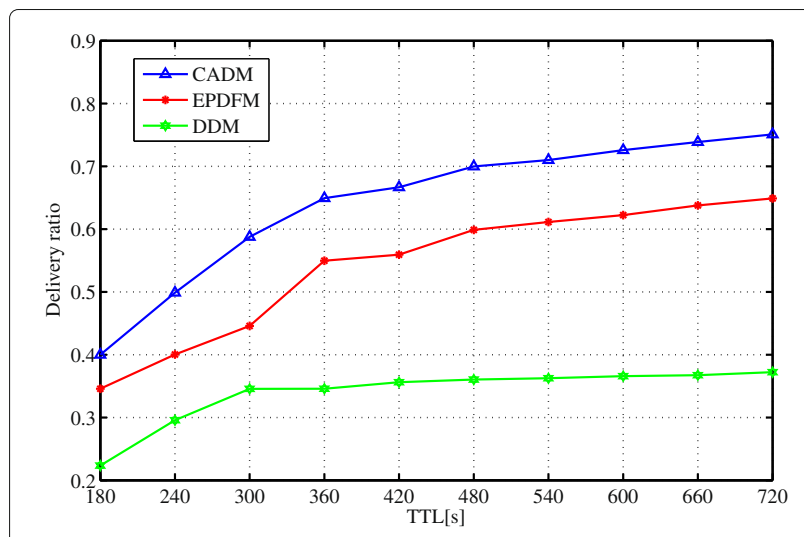

Fig. 6 The successful delivery ratio under various $T T L$ of data 


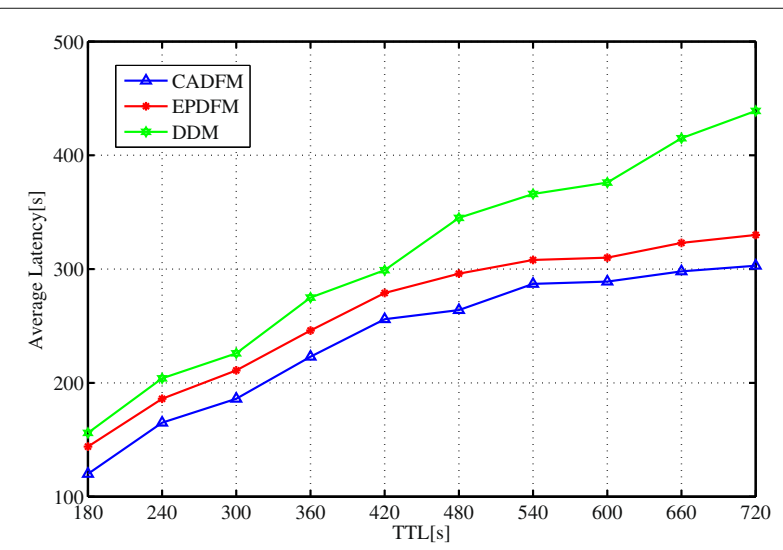

Fig. 7 The average delivery delay under various TTL of data

intelligent technologies such as machine learning and search technologies in edge network for smart transmission and IoT applications. For example, mobile edge computing will play a vital role in future connected vehicular system in which vehicles exchange information among each other via the help of mobile edge nodes available on the road. The information obtained from the knowledge layer, e.g., the trustworthiness between devices, could be used to facilitate the communications among IoT devices, to realize a trustworthy and reliable data transmission. Last but not the least, the proposed framework provides a new perspective in understanding the resource allocation problem in future cellular network, which is not available in the literature.

\section{Acknowledgements}

This work is partially supported by the National Natural Science Foundation of China $(61371097,61771082)$ and the Program for Innovation Team Building at Institutions of Higher Education in Chongqing (CXTDX201601020).

\section{Authors' contributions}

DW proposed the algorithm and wrote the paper. HW, RW, CZ, and JY derived the algorithm and performed the simulation work. All authors read and approved the final manuscript.

\section{Competing interests}

The authors declare that they have no competing interests.

\section{Publisher's Note}

Springer Nature remains neutral with regard to jurisdictional claims in published maps and institutional affiliations.

\footnotetext{
Author details

${ }^{1}$ College of Communication and Information Engineering, Chongqing University of Posts and Telecommunications, Chongwen Road, Chongqing, China. ${ }^{2}$ vivo Mobile Communication Co., Ltd., BBK Road, Guangdong, China. ${ }^{3}$ Electrical and Computer Engineering, University of Massachusetts Dartmouth, Old Westport Road, North Dartmouth, USA.
}

Received: 31 October 2017 Accepted: 24 April 2018 Published online: 25 May 2018

\section{References}

1. Visual Networking Index: Global Mobile Data Traffic Forecast Update, 2015-2020, Cisco Syst., San Jose, USA (2016). Downloaded from https:// www.cisco.com/c/en/us/solutions/collateral/service-provider/visualnetworking-index-vni/mobile-white-paper-c11-520862.html

2. JJ Yan, DP Wu, S Sanyal, RY Wang, Trust-oriented partner selection in D2D cooperative communications. IEEE Access. 5, 3444-3453 (2017). http:// doi.org/10.1109/ACCESS.2017.2671404

3. JJ Guo, JF Ma, XH Li, T Zhang, A situational awareness trust evolution model for mobile devices in D2D communication. IEEE Access. PP(99), 1-23 (2017). http://doi.org/10.1109/ACCESS.2017.2755058

4. Q Jiang, S Zeadally, JG Ma, DB He, Lightweight three-factor authentication and key agreement protocol for internet-integrated wireless sensor networks. IEEE Access. 5, 3376-3392 (2017). http://dx.doi. org/10.1109/ACCESS.2017.2673239

5. ZF Zhang, PR Zhang, D Liu, SH Sun, SRSM-based adaptive relay selection for D2D communications. IEEE Internet Things J. PP(99), 1-9 (2017). http://doi.org/10.1109/JIOT.2017.2749443

6. D Huang, T Xing, $\mathrm{H}$ Wu, Mobile cloud computing service models: a user-centric approach. IEEE Netw. 27(5), 6-11 (2013). http://dx.doi.org/10. 1109/MNET.2013.6616109

7. YB Miao, JF Ma, XM Liu, XH Li, ZG Liu, H Li, Practical attribute-based multikeyword search scheme in mobile crowdsourcing. IEEE Internet Things J. PP(99), 1-11 (2017). http://dx.doi.org/10.1109/JIOT.2017.2779124

8. F Bonomi, et al., Fog computing and its role in the internet of things, Edition of the Mcc Workshop on Mobile Cloud Computing. (ACM, 2012), pp. 13-16

9. JB Xiong, YY Zhang, X. Li, MW Lin, ZQ Yao, GQ Liu, RSE-PoW: A role symmetric encryption PoW scheme with authorized deduplication for multimedia data. Mob. Networks Appl. PP(99), 1-14 (2017). https://doi. org/10.1007/s11036-017-0975-x

10. M Peng, S Yan, K Zhang, CG Wang, Fog-computing-based radio access networks: issues and challenges. IEEE Network. 30(4), 46-53 (2016). http:// dx.doi.org/10.1109/MNET.2016.7513863

11. TR 45.820, Cellular system support for ultra-low complexity and low throughput Internet of (Things CloT). 3GPP. Downloaded from https:// portal.3gpp.org/desktopmodules/Specifications/SpecificationDetails. aspx?specificationld $=2719$

12. L Zhou, Mobile device-to-device video distribution: theory and application. ACM Trans. Multimed. Comput. Commun. Appl. (TOMM). 12(3), 1-23 (2016)

13. DP Wu, SS Si, SE Wu, RR Wang, Dynamic trust relationships aware data privacy protection in mobile crowd-sensing. IEEE Internet Things J. PP(99), 1-13 (2017). http://doi.org/10.1109/JIOT.2017.2768073

14. C Luo, S Guo, LT Yang, G Min, X Xie, Green communication in energy renewable wireless mesh networks: routing, rate control, and power allocation. IEEE Trans. Parallel Distrib. Syst. 25(12), 3211-3220 (2014). http://doi.org/10.1109/TPDS.2013.2297922

15. DP Wu, QR Liu, HG Wang, DL Wu, RY Wang, Socially aware energy-efficient mobile edge collaboration for video distribution. IEEE Trans. Multimedia. 19(10), 2197-2209 (2017). http://doi.org/10.1109/TMM.2017.2733300

16. DP Wu, JJ Yan, HG Wang, DL Wu, RY Wang, Social attribute aware incentive mechanism for device-to-device video distribution. IEEE Trans. Multimedia. 19(8), 1908-1920 (2017). http://dx.doi.org/10.1109/TMM. 2017.2692648

17. W Wang, C Li, in Proc. 2012 IEEE 4th Int. Conf. CASoN. A Core-based community detection algorithm for networks (IEEE, 2012), pp. 20-25

18. DP Wu, J He, HG Wang, CG Wang, RR Wang, A hierarchical packet forwarding mechanism for energy harvesting wireless sensor networks. IEEE Commun. Mag. 53(8), 92-98 (2015). http://doi.org/10.1109/MCOM. 2015.7180514

19. ZF Zhang, L Wang, J Zhang, Energy efficiency of D2D multi-user cooperation. Sensors. 17(4), 697-709 (2017). http://doi.org/10.3390/ s17040697

20. D Watts, S Strogatz, Collective dynamics of small-world networks. Nature. 393, 440-442 (1998). http://doi.org/10.1038/30918

21. R. Bond, C Fariss, J Jones, A 61-million-person experiment in social influence and political mobilization. Nature. 487, 295-298 (2012). http:// doi.org/10.1038/nature11421

22. T Li, C Wu, P Hui, Social-aware D2D communications: qualitative insights and quantitative analysis. IEEE Commun. Mag. 52(6), 150-158 (2014). http://doi.org/10.1109/MCOM.2014.6829957

23. Y Zhang, E Pan, Social network aware device-to-device communication in wireless networks. IEEE Trans. Wirel. Commun. 14(1), 177-190 (2014). http://doi.org/10.1109/TWC.2014.2334661 
24. L Wang, L Liu, X Cao, DL Wu, RY Wang, Sociality-aware resource allocation for device-to-device communications in cellular networks. IET Commun. 9(3), 342-349 (2015). http://doi.org/10.1049/iet-com.2014.0436

25. D Wu, L Zhou, Y Cai, Social-aware rate based content sharing mode selection for D2D content sharing scenarios. IEEE Trans. Multimedia. 19(11), 2571-2582 (2017). http://doi.org/10.1109/TMM.2017.2700621

26. L Zhou, R Hu, Y Qian, HH Chen, Energy-spectrum efficiency tradeoff for video streaming over mobile ad hoc networks. IEEE J. Sel. Areas Commun. 31(5), 981-991 (2013). http://doi.org/10.1109/JSAC.2013.130516

27. W Saad, H Zhu, M Debbah, in Proc. 28rd INFOCOM. Coalitional games for distributed collaborative spectrum sensing in cognitive radio networks (IEEE, 2009), pp. 2114-2122

28. T Karagiannis, L Boudec, J Vojnovic, Efficiency resource allocation for device-to-device underlay communication systems: a reverse iterative combinatorial auction based approach. IEEE J. Sel. Areas Commun. 31(9), 348-358 (2013). http://doi.org/10.1109/JSAC.2013.SUP.0513031

29. C Xu, L Song, Z Han, D Li, in Proc.GLOBECOM. Resource allocation using a reverse iterative combinatorial auction for device-to-device underlay cellular networks, (IEEE, 2012), pp. 4542-4547

30. J Tian, HX Zhang, D Wu, D Yuan, Interference-aware cross-layer design for distributed video transmission in wireless networks. IEEE Trans. Circuits Syst. Video Technol. 26(5), 978-991 (2016). http://doi.org/10.1109/TCSVT. 2015.2430611

31. T Karagiannis, L Boudec, J Vojnovic, Power law and exponential decay of intercontact times between mobile devices. IEEE Trans. Mob.Comput. 9(10), 1377-1390 (2010). http://doi.org/10.1109/TMC.2010.99

32. A Chaint, P Hui, J Crowcroft, Impact of human mobility on opportunistic forwarding algorithms. IEEE Trans. Mob. Comput. 6(6), 606-620 (2007). http://doi.org/10.1109/TMC.2007.1060

33. J Scott, R Gass, J Crowcroft, P Hui, C Diot, A Chaintreau, CRAWDAD trace cambridge/haggle/imote/infocom2006 (v. 2009-05-29) (2009).

Downloaded from http://crawdad.cs.dartmouth.edu/cambridge/haggle/ imote/infocom2006

\section{Submit your manuscript to a SpringerOpen ${ }^{\circ}$ journal and benefit from:}

- Convenient online submission

- Rigorous peer review

- Open access: articles freely available online

- High visibility within the field

- Retaining the copyright to your article

Submit your next manuscript at $>$ springeropen.com 